\title{
Bar in Questionnaires
}

\author{
Thomas Vougiouklis \\ Democritus University of Thrace, Alexandroupolis, Greece \\ Penelope Kambakis-Vougiouklis \\ Democritus University of Thrace, Komotini, Greece
}

\begin{abstract}
Vougiouklis \& Vougiouklis have proposed the replacement of Likert scales, usually used in questionnaires, with a bar. With this proposal a discrete situation is replaced by a fuzzy one. There are identified certain advantages concerning the use of the bar as compared to that of a scale during both the stages of filling-in as well as processing a questionnaire. The main advantage associated with business research requirements is the fact that it is expected to be much quicker to fill in and much easier to explain to participants. The bar provides the potential for different types of processing Likert scales cannot. Therefore the researchers are allowed to ascertain that the given answers follow not only the already suggested and used Gauss distribution but also a parabola distribution as it will be suggested in present paper, and which is expected to give the researcher the opportunity to "correct" this tendency. Therefore, a possibility of choosing amongst a number of alternatives is offered, by utilizing fuzzy logic in the same way as it has already been done in industry and combining mathematical models with multivalued operations.
\end{abstract}

Keywords: bar, Likert scale, fuzzy logic, questionnaire, mathematical models, hyperstructures

\section{Theoretical Background}

Mathematical models are often used in almost every field of empirical research in order to reinforce the reliability of each individual research. Moreover, mathematicalisation of a problem could make its results recognizable and comparable with other results. This is because representing an actual research object or a phenomenon with numbers and figures or graphs might be the simplest and the most recognizable way of reading the actual results.

In every empirical research three main stages could be normally identified: design, implementation, and processing of the results. Main tools in an empirical research include the questionnaire where Likert scales are normally and widely used. Likert scales are often used to measure respondents' attitudes by asking the extent to which they agree or disagree with a particular question or statement. Likert scales may seem easy to analyze but there are important issues a data analyst should consider. More specifically, there are certain shortcomings usually identified in this type of scales and they include the range of the scale which each time is upon the researcher to decide as it is not standard how many different subdivisions, or grades, should be used. Moreover, this is not an easy job to accomplish as it is quite different to have three or four or five subdivisions since there are certain problems to overcome in each case. Such problems include lack of a medium choice in a four-grade

Thomas Vougiouklis, Professor of Mathematics, Head, Department of Education, Democritus University of Thrace.

Penelope Kambakis-Vougiouklis, Associate Professor of Linguistics, Department of Greek, Democritus University of Thrace.

Correspondence concerning this article should be addressed to Thomas Vougiouklis, School of Education, Democritus University of Thrace, Nea Hili, 681 00, Alexandroupolis, Greece. E-mail: tvougiou@eled.duth.gr. 
scale. Another shortcoming of typical Lickert scales is the difficulty of verbally refining the difference between different subdivisions and makes them clear to the participants, especially to less sophisticated ones. This is not an easy process, as many researchers report that it takes their subjects more time to comprehend what each subdivision represents on their scale rather than accomplish the actual test. Such a problem is of course not purely linguistic but it involves a number of different factors such as social and psychological. Finally, in the stage of processing the results, the researchers will have only one possibility of working them out, the one they decided to establish when initially designing the experiment. Such a decision, though, might deprive the researchers of the possibility to explore other parameters which might crop up in the process, or even try different subdivisions for either a more accurate calculation or in order to make their results comparable with somebody else's who has used different scale.

In order to eliminate such shortcomings, we propose the substitution of Likert scales with the bar, as suggested by Vougiouklis \& Vougiouklis (Kambaki-Vougioukli \& Vougiouklis, 2008; Lygeros, 2009; Kambaki-Vougioukli, Karakos, Lygeros, \& Vougiouklis, 2011; Vougiouklis \& Kambaki-Vougioukli, 2011). This substitution makes things simpler and easier for both the subjects of an empirical research and the researcher, either at the stage of designing or that of results processing, because it is really flexible. Moreover, the application of the bar opens a window towards the use of fuzzy sets in the whole procedure of empirical research, activating in this way more recent findings from different sciences, as well.

More specifically, the following was proposed (Kambaki-Vougioukli \& Vougiouklis, 2008):

In every question, substitute the Likert scale with the "bar" whose poles are defined with " 0 " on the left and " 1 " on the right:

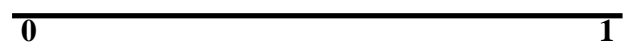

The subjects/participants are asked, instead of deciding and checking a specific grade on the scale, to cut the bar at any point they feel best expresses their answer to the specific question.

In Vougiouklis and Kambakis-Vougiouklis (2011), it was suggested that the appropriate length of the bar is approximately $6.18 \mathrm{~cm}$, or $6.2 \mathrm{~cm}$, following the golden ration on the well-known length of $10 \mathrm{~cm}$. More precisely it is considered that the average participant is over-familiarized with the $10 \mathrm{~cm}$ length and an original suggestion might be the use of the golden ratio, suggested by the ancient Greeks. The golden ratio has not been used in language research and it might have the potential to be a useful tool in empirical research.

\section{Comparing Scales With the Bar}

Grading a variant depends both on its actual nature as well as in the researcher's judgment. Apparently, there are certain scales which are very often used, for example the five/grade one with choices such as:

"I totally disagree", "I disagree", "I am between", "I agree", and "I absolutely agree".

This type of scale is characterized by necessary elements-rules which normally identify such scales. More specifically, they usually start from the absolutely negative and end to the absolutely positive, as in the example above, or vice-versa. However, the most serious problem constitutes the discrimination of the limits of the actual partition. This problem of discrimination of the different categories concerns both the researcher and the subjects/participants and many times may result in frustration, especially on the part of the latter. It is a common practice to spend a lot of time in order to clarify questions posed by the participants. Furthermore, it might be possible for a participant to be able to identify the difference in one or most of the questions but not in 
every question, which is the optimum in any research. Consequently, the participants have to adapt themselves to the specific type of categorization in every single question - a task not at all easy and pleasant, which might affect the reliability of the specific results or, even worse, it might yield misleading ones. In such a situation it might prove to be a real hazard if it concerns issues such as health or serious financial investments and important business projects heavily depending on market research. We would not exaggerate, if we suggested that researchers should include another parameter, that of the reliability of the answers, which is connected to the level of the subjects' familiarization to the actual type of evaluation of the questions. Researchers, trying to avoid some undesirable side-effects such as the risk of coming up with a concentration of the majority of the answers in the middle of the scale, choose a scale with no medium choice such as a 4-point scale. Clearly the kind of research will always decide the type of scale to be used, however if the researchers want to investigate some other parameters they had not provided for well in advance, or if they want to view some aspects from a different point of view, they will have to re-apply the test with a different scale. In this case, though, they will have to begin the process of familiarization of her/his subjects with the new categorization. Consequently, in every case, the problem is the establishment and evaluation of the "unit".

The important subject of the linguistic parameter of the scales and its correlation to the fuzzy theory has been widely investigated by Zadeh (1965), the founder of the fuzzy theory. Zadeh has characterized the related variable "linguistic variable" accepting it in this way as the generating cause of fuzzy sets, something extremely important for linguistics. Yet, the linguistic escalation of the question parameter is not an easy job to accomplish (Kambaki-Vougioukli, 2009), as it depends upon factors other than purely linguistic, including all psychological, educational, economic and social status, age, gender and/or even language of the subjects. Consequently, in the normally used questionnaires with Likert scales, it is rather risky to claim that the grades of the scale are clearly pinpointed so that to be completely comprehensible by all subjects. For example, in a scale where:

$$
0=\text { completely unsure, } 1=\text { rather unsure, } 2=\text { almost sure, and } 3=\text { absolutely sure }
$$

We can never be sure whether everybody distinguishes between 3 and 2 or 0 and 1 in the same way. Even more so, some subjects would like something in the middle or, even, something between 1 and 2 or 2 and 3 etc., i.e., a more detailed escalation. It is as if you give people a flight of stairs the height of which is good only for people who are, say, $180 \mathrm{~cm}$ tall while the shorter or younger ones will have trouble using them.

In Vougiouklis and Kambaki-Vougioukli (2011), it is claimed that there is no need to demand from our subjects to try to distinguish the difference between the grades of a scale, an attempt which is tough anyway and might very well be in vain. Furthermore, we are sure that no special training will be necessary for our subjects so as to enable them to understand how to cut the bar proposed, as they can cut it intuitively, without any verbal processing. Such a process could be compared to somebody in a wheelchair trying to go up an inclined plane using a ramp rather than a flight of stairs. Consequently, the psychological factor could be easily controlled as the subject is asked to cut the bar 01 based only on pure intuition, which actually defines the most accurate point that specific moment the decision is made. The bar, in other words, gives access to a fuzzy attitude since it requires a mapping in the space 01 instead of a discreet answer 0 or 1 .

Another issue that it is worth referring to is the following: If subjects are used to filling in questionnaires using Likert scales, then they are expected to have created in their mind an abstract scale of 3, 4, or 4 grades, or, even, of 10 or 100 grades or steps, by means of which they evaluate the questions posed. It is widely accepted that normally 10 is used for grading or marking while 100 is used for percentages; then, obviously, the percent 
answer is closer to that of the bar, since the 100 -grade escalation of the short bar is closer to that of a continuum than the 10-grade one. However there is still one indication in the middle for which the least decisive or reluctant one are expected to go for! The suggestion that we should establish equal spaces along the bar using mill metric paper is inappropriate as it actually affects the subjects' answers guiding them towards certain points along the bar.

Lygeros (2009 p. 1055) pointed out that "the Vougiouklis \& Vougiouklis innovation concerning the substitution of the scale with the bar is originated from the contention between continuum and discrete".

\section{Advantages of the Bar in the Processing of the Results}

Processing questionnaires using the bar gives the initiative to the researchers who have the chance to "escalate" the answers without having to decide in advance how many grades they will finally need $3,4, \ldots, 10$ in order to be able to identify the parameters and clarify the differences between the grades. More importantly, they offer the flexibility of establishing balanced or imbalanced scales according to the needs of the specific research each time. In other words, after all the filled in questionnaires have been collected, the researchers will be able to access and process them in more than one way without having to repeat the test. In this way, the subjects will be protected from going through a time consuming and rather unpleasant process but even more avoiding to risk the reliability of the results. This is because sitting the same test again, may involve the risk of the specific subjects' familiarization with the whole process and consequently affecting the reliability of the research itself. More clearly, a danger is involved of eliciting answers that are not spontaneous but "desired" because the participants might have "guessed" what the researchers' expects. The use of the bar minimizes such risks and reinforces the objectivity of it as it gives more space to mathematical processing of the results.

One of the main characteristics of applied mathematics is the ability of different approaches, the ability of simplification of the form. That is why linear models are preferred and we tend to change continuum into discrete and vice-versa. Consequently the bar offers the possibility of accurate processing which is the optimum for the researcher: from discrete into continuous and, even more, from single valued into multivalued or fuzzy (Davvaz, 2003, 2013; Davvaz \& Leoreanu, 2007; Kambaki-Vougioukli et al., 2011; Kambaki-Vougioukli \& Vougiouklis, 2008; Vougiouklis, 1994, 2008; Vougiouklis \& Kambaki, 2008).

The use of the multivalued maps and the hyperstructure theory which is closely related with fuzzy set theory (Kambaki-Vougioukli, 2009; Lygeros, 2009; Vougiouklis, 2008) could be of assistance to both data processing and, amongst others, language teaching (Vougiouklis \& Kambaki, 2008). It would be a very good idea to make an extra evaluation of our proposal by asking participants who have filled in questionnaires using Likert scales in the past, to specify whether the bar or the other type of scale makes things easier for them. Or even have a simultaneous use of both methods and ask for an immediate evaluation.

We thought that such an exact and stable length gives it the advantage of an easy and accurate comparability among different researchers and researches anywhere in the world. Not to mention the burden of having to define and then explain to the participants all the fine differences between terms or phrases used. More specifically, if we need to choose from an escalation including very good, good, fairly good, almost good, not really good, not good, not good enough, rather bad, not very bad, etc., how can we be sure that we can explain the differences accurately and make each of them completely comprehensible and easily distinguished from the other relative choices to all our participants? In the case of the bar, however, we leave this decision upon the participants, even without having to explain it verbally. 
A really difficult problem for researchers to solve is, if they have to change a, say, 5-grade scale such as "I totally disagree", "I disagree", "I am between", "I agree”, "I absolutely agree”, into a 6-grade one. The researchers then will have to find a new word or phrase to put into somewhere between the words or phrases in use and moreover to redefine the limits and the meanings of these words (or phrases).

Certain applications of the suggested method for questionnaire processing via the bar divided into five equal area-spaces according to Gauss distribution, applied at Democritus University, in the frame of evaluation have come up with very interesting results. Moreover, computerizing the questionnaires using the bar makes it more attractive to the students, who seem to prefer the bar instead of the scale with an overwhelming majority of the bar, $93 \%$, as compared to Likert scale.

\section{Data Processing: The Parabolic Case}

Questionnaire procession using the bar gives the initiative to the researcher, who has the chance to "escalate" the answers without having to decide in advance how many grades there will be finally needed so as to be able to identify the parameters and clarify the differences between the grades. Even more so, there is a good degree of flexibility in establishing balanced or imbalanced scales taking into consideration the needs of the specific research each time. More specifically, after the researcher has collected the filled-in questionnaires, he/she will be able to process and access them in numerous ways without having to repeat the test putting the subjects in a new time consuming process and risking the reliability of results. Using the bar minimizes such risks and, more importantly, reinforces the objectivity of it as it gives more space to mathematical processing of the results.

Now we come to the final stage, that of the processing the result. The procedure to be followed is exactly the same as the one followed with any Likert scale. That is to say, we can divide the bar in as many equal spaces as we wish. One could ask why should we go through the bar procedure then? The answer is that our bar gives a potential for different types of processing not provided by the Likert scales. For example, let us suppose that the researcher finds out that the given answers follow the Gauss distribution and wishes to "correct" this tendency (Mathioudakis, 2009, 2013; Mouti, 2011; Vougiouklis \& Kambaki-Vougioukli, 2011).

We remind that we had analogous classes using the Gauss distribution which are as follows (Vougiouklis \& Kambaki-Vougioukli, 2011), in mm:

For 3 classes with limit values $0-27-35-62$

For 4 classes with limit values $0-25-31-37-62$

For 5 classes with limit values $0-24-29-33-38-62$

For 6 classes with limit values $0-22-27-31-35-40-62$

For 7 classes with limit values $0-21.5-26.0-29.5-32.5-36.0-40.5-62$

The case we now set as the main point of our paper, is the following: We suppose that the researcher consider that the answers are decreasing, say, for example, for psychological reasons, from the left to the right side of the segment $[0,1]$. One of the models to represent the situation is the decreasing parabola $x=1-y^{2}$. In order to correct (normalize) the results we can divide the continuum $[0,1]$ into equal-area spaces according to the above decreasing parabola distribution. This can be done by dividing the segment $[0,62]$ in equal-area spaces as follows:

For the increasing low parabola, in its canonical form: $x=y^{2}$, we have the following segments, in $\mathrm{mm}$ : 
For 3 classes with limit values $0-43-54-62$

For 4 classes with limit values $0-39-49-56-62$

For 5 classes with limit values $0-36-46-52-58-62$

For 6 classes with limit values $0-34-43-49-54-58-62$

For 7 classes with limit values $0-32-41-47-51-55-59-62$

A second case is the increasing upper parabola which is represented by $1-y=(1-x)^{2}$, and it is symmetric to the above with respect to the point $(0.5,0.5)$. Then we divide the segment $[0,62]$ in equal-area spaces as follows, in mm:

For 3 classes with limit values $0-32-48-62$

For 4 classes with limit values $0-27-40-51-62$

For 5 classes with limit values $0-24-35-45-54-62$

For 6 classes with limit values $0-22-32-40-48-55-62$

For 7 classes with limit values $0-20-30-37-44-50-56-62$

The corresponding decreasing cases are symmetric, to line $x=31$, therefore the results are symmetric. More precisely we have the cases:

For the decreasing low parabola, in its canonical form: $y=(1-x)^{2}$, we have the following segments, in mm:

For 3 classes with limit values $0-8-19-62$

For 4 classes with limit values $0-6-13-23-62$

For 5 classes with limit values $0-4-10-16-26-62$

For 6 classes with limit values $0-4-8-13-19-28-62$

For 7 classes with limit values $0-3-7-11-15-21-30-62$

The second case is the decreasing upper parabola which is represented by $1-y=x^{2}$, we have the following segments, in $\mathrm{mm}$ :

For 3 classes with limit values $0-14-30-62$

For 4 classes with limit values $0-11-22-35-62$

For 5 classes with limit values $0-8-17-27-38-62$

For 6 classes with limit values $0-7-14-22-30-40-62$

For 7 classes with limit values $0-6-12-18-25-32-42-62$

\section{Conclusions}

Moreover, computerizing the questionnaires using the bar it becomes more attractive, the students prefer the bar instead of the scale. Concerning the latter, there was an overwhelming superiority of the bar, $93 \%$, as compared to Likert scale.

From what has been discussed so far as well based on the results of the so far experimental applications, it might be clear that using the bar in questionnaires provides an extremely useful advantage closely related to business research. This is the immediate, quick, and accurate response of both the parts involved in such a process: (1) the participants, who can fill in the questionnaire in an easy and comfortable way and give spontaneous answers without much thought and no insecurity; and (2) the researchers, who do not have to go through complicated and complex explanations and still not being sure that they have clarified their purpose well enough so as to have the desired feedback. Moreover and on the researchers' part, they are offered a 
number of opportunities as to elaborate on the processing of the collected questionnaires and to process and access them in numerous ways without having to repeat the test. The use of the bar minimizes the risks and, more importantly, reinforces the objectivity of the experiment as it gives more space to mathematical processing of the results. The classes of mathematics which are used vary from fuzzy sets to hyperstructures. The researchers are able to face new problems appearing in business, in industry, and in social sciences.

Moreover, computerizing the questionnaires using the bar makes it more attractive, and, as mentioned above, it seems to be an overwhelming preference of the bar rather than Likert scales among students.

\section{References}

Davvaz, B. (2003). A brief survey of the theory of $H_{v}$-structures (pp. 39-70). Samothraki, Greece: Spanidis Press.

Davvaz, B. (2013). Polygroup theory and related systems. Hackensack: World Scientific.

Davvaz, B., \& Leoreanu, V. (2007). Hyperring theory and applications. Palm Harbor, Fla, USA: International Academic Press.

Kambaki-Vougioukli, P. (2009). Language and mathematical models. Thessaloniki, Greek: Kyriakidis Press.

Kambaki-Vougioukli, P. (2013). Bar in SILL questionnaire for multiple results processing: Users' frequency and confidence, stead of scale. Sino-US English Teaching, 10(3), 184-199.

Kambaki-Vougioukli, P., \& Vougiouklis, T. (2008). Bar instead of scale. Ratio Sociologica, 3, 49-56.

Kambaki-Vougioukli, P., Karakos, A., Lygeros, N., \& Vougiouklis, T. (2011). Fuzzy instead of discrete. Annals of Fuzzy Mathematics and Informatics (AFMI), 2(1), 81-89.

Lygeros, N. (2009). Interrogations fondamentales sur la méthodologie du questionnaire. Retrieved from http://www.lygeros.org/

Mathioudakis, N. (2009). The fuzzy areas of accuracy and confidence while guessing the idiosyncratic vocabulary of Nikos Kazantzakis' "O $\triangle \mathrm{Y} \Sigma E I A "$. Proceedings from the 9th International Conference on Greek Linguistics (ICGL9). Chicago-Illinois.

Mathioudakis, N. (2013). The fuzzy areas of guessing or inferencing the idiosyncratic vocabulary of Nikos Kazantzakis' ODYSSEY (O $\triangle$ Y $\Sigma$ EIA). Proceedings from 11th International Conference on Greek Linguistics (ICGL 11). Rhodes, Greece.

Mouti, A. (2011). Investigating cognitive style as a factor affecting language test performance and confidence. Retrieved from http://phdtheses.ekt.gr/eadd/handle/10442/28264

Nikolaidou, P., \& Vougiouklis, T. (2012). $\mathrm{H}_{\mathrm{v}}$-structures and the bar in questionnaires. Italian J. Pure Applied Math, 29, $341-350$.

Vougiouklis, T. (1994). Hyperstructures and their representations. Florida: Hadronic Press.

Vougiouklis, T. (2008). $\partial$-operations and $\mathrm{H}_{\mathrm{v}}$-fields. Acta Mathematica Sinica, 24(7), 1067-1078.

Vougiouklis, T., \& Kambaki, P. (2008). Algebraic models in applied research. Jordan J. Math. Statistics, 1(1), 78-87.

Vougiouklis, T., \& Kambaki-Vougioukli, P. (2011). On the use of the bar. China-USA Business Review, 10(6), 484-489.

Zadeh, L. A. (1965). Fuzzy sets. Information and Control, 12(2), 94-102. 